\title{
Global Revascularization by Percutaneous Intervention
}

\section{Sohrabuzzaman APIVI; Islam R, Towhiduzzaman-M}

Percutaneous intervention is a well utilized and widely practiced procedure for opening up of clogged blood vessels. It is used from small to large arteries, native to graft vessels and shunts. We have done a large number of cases in our center which includes coronary arteries, bypass graft vessels, renal arteries and arteries of the upper and lower limbs. The total number of procedures is two thousand in last two years. Among these twelve hundred were complex coronary cases and a few were peripheral cases. We are presenting some of our interesting cases here. 